\title{
ALGEBRAIC GROUPS WITH FEW SUBGROUPS
}

\author{
SKIP GARIBALDI AND PHILIPPE GILLE
}

\begin{abstract}
Every semisimple linear algebraic group over a field $F$ contains nontrivial connected subgroups, namely maximal tori. In the early $1990 \mathrm{~s}$, J. Tits proved that some groups of type $E_{8}$ have no others. We give a simpler proof of his result, prove that some groups of type ${ }^{3} D_{4}$ and ${ }^{6} D_{4}$ have no nontrivial connected subgroups, and give partial results for types ${ }^{1} E_{6}$ and $E_{7}$. Our result for ${ }^{3} D_{4}$ uses a general theorem on the indexes of Tits algebras which is of independent interest.
\end{abstract}

\section{Contents}

1. Introduction 1

2. First steps 3

3. Splitting fields of tori 4

4. Global fields 6

5. Versal groups $\quad 7$

6. Rost invariant and adjoint groups 10

7. Groups of type $E_{8} \quad 12$

8. Tits algebras 13

9. Weyl group of $E_{6} \quad 14$

10. Groups of type ${ }^{1} E_{6} \quad 16$

11. Groups of type $E_{7} \quad 17$

12. Maximal indexes of Tits algebras 20

13. Groups of type ${ }^{3} D_{4}$ or ${ }^{6} D_{4} \quad 22$

References $\quad 24$

\section{INTRODUCTION}

In [Ti 92b], Jacques Tits proved that there exist algebraic groups of type $E_{8}$ that have very few subgroups. Specifically, he proved that they are almost abelian, ${ }^{1}$ i.e., they have no proper, nonzero closed connected subgroups other than maximal tori. Tits also gave a partial result towards the existence of almost abelian groups of type $E_{6}$ in [Ti 92a].

2000 Mathematics Subject Classification. 20G15.

Version of May 13, 2008.

${ }^{1}$ This term was suggested by A. Premet. 
Since his work, new tools have appeared, making the problem of construction almost abelian groups easier. We give a simpler proof of his result for groups of type $E_{8}(\S 7)$, extend his result for groups of type $E_{6}$ (Prop. 10.1), and prove a similar partial result for groups of type $E_{7}$ (Prop. 11.1). Roughly speaking, for groups of types ${ }^{1} E_{6}$ and $E_{7}$, we settle the question of existence of reductive subgroups up to prime-to- $p$ extensions of the base field for each prime $p$.

For groups of type $D_{4}$, we can do more. We prove:

Theorem 1.1. If $G$ is a superversal group of type ${ }^{3} D_{4}$ or ${ }^{6} D_{4}$ over a field of characteristic $\neq 2,3$, then $G$ is almost abelian.

This answers a question by A. Premet and is proved in $\S 13$. The proof uses a result about maximal indexes of Tits algebras for groups of outer type (Th. 12.1), which is interesting by itself.

A superversal group is roughly speaking the most general group of the given type, see Definition 5.2 below. For global fields, such examples do not occur, see section 4 .

We remark that groups of type $G_{2}$ or $F_{4}$ are never almost abelian, because the long roots generate a subgroup of type $A_{2}$ or $D_{4}$ respectively. Thus we have treated the question of existence of reductive subgroups for all exceptional groups, except that for types $E_{6}$ and $E_{7}$ we have only done so up to prime-to- $p$ extensions of the base field for each prime $p$ and for inner forms.

The idea behind Tits's proofs and ours is to find groups that require a field extension of large degree to split them. We combine his methods with the recent computations of torsion indexes by Totaro [To] and the Rost invariant defined in [Mer 03].

Notation and conventions. The groups we discuss are linear algebraic groups in the sense of [Bo]. We write $\mu_{n}$ for the group whose $F$-points are the $n$-th roots of unity in $F$. We define "reductive group" to include the hypothesis that the group is connected.

For a field $F$, we write $\operatorname{Gal}(F)$ for its absolute Galois group, i.e., the Galois group of a separable closure $F_{\text {sep }}$ of $F$ over $F$. If $\operatorname{Gal}(F)$ is a $p$-group (i.e., if every finite separable extension of $F$ is of dimension a power of $p$ ), then we say that $F$ is $p$-special. The subfield $F_{p}$ of $F_{\text {sep }}$ consisting of elements fixed by a $p$-Sylow subgroup of $\operatorname{Gal}(F)$ is $p$-special. We call $F_{p}$ a co-p-closure of $F$.

For a central simple $F$-algebra $D$, we write $\mathrm{SL}_{1}(D)$ for the algebraic groups whose $F$-points are the norm 1 elements of $D$.

We use extensively the notion of Tits index of a semisimple group as defined in [Ti66]. The list of possible indexes is given in that paper and also in [Sp, pp. 320, 321]. (There is a typo in index \#14 in Springer's table; it needs to have an additional circle darkened. His Prop. 17.8.2 is correct.) 


\section{First Steps}

Let $G$ be a semisimple algebraic group over a field $F$ of characteristic zero. By the type of $G$, we mean its Killing-Cartan type over an algebraic closure of $F$.

2.1. The property of being almost abelian is an invariant of isogeny classes. That is, for every central isogeny $G \rightarrow G^{\prime}$, the group $G$ is almost abelian if and only if $G^{\prime}$ is.

2.2. If $G$ is isotropic, then $G$ is not almost abelian. Indeed, if $G$ is isotropic then it contains a rank 1 split torus over $F$, and when $G$ has absolute rank 2 this suffices to show that $G$ is not almost abelian. If $G$ is isotropic and of type $A_{1}$, then it is isogenous to $\mathrm{SL}_{2}$ and so has a Borel subgroup defined over $F$.

2.3. Our general plan is the following. For a specific semisimple group $G$ over a field $F$, we prove three statements:

(1) $G$ is $F$-anisotropic.

(2) $G$ does not contain any $F$-defined proper semisimple subgroups of the same absolute rank as $G$.

(3) Every nonzero $F$-torus in $G$ is maximal.

This will show that every nonzero proper (connected) reductive subgroup of $G$ is a maximal torus. Indeed, let $H$ be a nonzero proper reductive subgroup of $G$. By (3), $H$ has the same absolute rank as $G$. Again by (3), the central torus of $H$ is either trivial or all of $H$, i.e., $H$ is semisimple (excluded by (2)) or a maximal torus, as claimed.

In order to prove (2), we note that an $F$-defined semisimple subgroup $H$ of $G$ of the same absolute rank contains - and so is normalized by - a maximal torus of $G$, hence $H$ is generated over an algebraic closure of $F$ by root subgroups of $G$ [Bo, 13.20]. That is, the roots of $H$ are a subsystem of the roots of $G$, and $H$ is of one of the the types described in [BdS] or [Dy, Th. 5.3], even though we do not assume that $F$ has characteristic zero.

In practice, item (3) is the difficult one. For this, our work is cut in half by the following elementary observation.

Symmetry Lemma 2.4. Let $G$ be a reductive algebraic group of absolute rank $n$. If $G$ contains an $F$-torus of rank $m$, then it contains an $F$-torus of rank $n-m$.

Proof. Let $S$ be an $F$-torus in $G$ of rank $m$. It is contained in a maximal $F$-torus $T$ of rank $n$. We switch to the dual category of finitely generated abelian groups with a continuous action by $\operatorname{Gal}(F)$. The natural projection $T^{*} \rightarrow S^{*}$ splits over $\mathbb{Q}$, i.e., there is a map $f: S^{*} \otimes \mathbb{Q} \rightarrow T^{*} \otimes \mathbb{Q}$ that is compatible with the Galois action and such that $\left.f(\beta)\right|_{S}=\beta$ for all $\beta \in S^{*}$. Set $S_{0}^{\prime}$ to be the intersection of $f\left(S^{*} \otimes \mathbb{Q}\right)$ with $T^{*}$ and

$$
S^{\prime}:=\left(\cap_{\alpha \in S_{0}^{\prime}} \operatorname{ker} \alpha\right)^{0} .
$$




\begin{tabular}{|c|c|}
\hline type & torsion primes \\
\hline$A_{n}(n \geq 1), C_{n}(n \geq 2)$ & none \\
$B_{n}(n \geq 3), D_{n}(n \geq 4), G_{2}$ & 2 \\
$F_{4}, E_{6}, E_{7}$ & 2,3 \\
$E_{8}$ & $2,3,5$ \\
\hline
\end{tabular}

Then $S^{\prime}$ is an $F$-subtorus of $T$ such that $T=S . S^{\prime}$ and $S \cap S^{\prime}$ is finite; it is the desired torus.

2.5. Suppose now that $F$ is perfect or the characteristic of $F$ is not a torsion prime for $G$ as listed in Table 2. Then every closed, connected unipotent subgroup of $G$ is contained in the radical of a (proper) parabolic subgroup by [BoTi, 3.7] and [Ti 87, 2.6], hence $G$ is isotropic. That is, if $G$ is anisotropic, then every closed, connected subgroup is reductive. Arguing as in 2.3 allows us to conclude that $G$ is almost abelian.

2.6. We illustrate the material above by classifying the almost abelian groups of type $A_{1} \times A_{1} \times A_{1}$. Every group of this type is isogenous to a transfer $R_{L / F}\left(\mathrm{SL}_{1}(Q)\right)$, where $L$ is a cubic étale $F$-algebra and $Q$ is a quaternion $L$-algebra. If $L$ is not a field, then such a group is obviously not almost abelian, because it contains an $F$-defined subgroup of type $A_{1}$. In case $L$ is a field, we have:

Proposition 2.7 ("type $A_{1} \times A_{1} \times A_{1}$ "). With the notation of 2.6, if $L$ is a field, then the following are equivalent:

(1) $R_{L / F}\left(\mathrm{SL}_{1}(Q)\right)$ is almost abelian.

(2) $Q$ is a division algebra and does not contain a quadratic field extension of $F$.

(3) $Q$ is a division algebra and the corestriction $\operatorname{cor}_{L / F}(Q)$ is not split.

Proof. Suppose (2) holds. As $Q$ is division, $R_{L / F}\left(\mathrm{SL}_{1}(Q)\right)$ is anisotropic. The group has no proper semisimple subgroups of rank 3 over an algebraic closure, so it cannot have one over $F$. Finally, if $R_{L / F}\left(\mathrm{SL}_{1}(Q)\right)$ contains a rank $1 F$-torus, then there is a quadratic extension of $F$ splitting $Q$, which is impossible by (2). By the Symmetry Lemma, $R_{L / F}\left(\mathrm{SL}_{1}(Q)\right)$ also has no rank 2 tori. Applying 2.3, we conclude that $R_{L / F}(\mathrm{SL}(Q))$ is almost abelian.

If (2) fails, i.e., if $Q$ is not division or does contain a quadratic extension $K$ of $F$, then $R_{L / F}\left(\mathrm{SL}_{1}(Q)\right)$ is isotropic or contains a rank $1 F$-torus respectively, hence is not almost abelian. That is, (1) implies (2).

The equivalence of (2) and (3) is [KMRT, 43.9].

\section{SPlitTing FiELDS OF TORI}

We recall the well-known divisibility bounds on finite subgroups of $\mathrm{GL}_{n}(\mathbb{Z})$ and $\mathrm{GL}_{n}(\mathbb{Q})$ given by Table 3. (We remark that every finite subgroup of 
$\mathrm{GL}_{n}(\mathbb{Q})$ is conjugate to a finite subgroup in $\mathrm{GL}_{n}(\mathbb{Z})$ by [Se 92 , p. 124], so the bounds are the same whether one takes $\mathbb{Z}$ or $\mathbb{Q}$.)

\begin{tabular}{|l|cc|}
\hline$n$ & finite subgroups of $\mathrm{GL}_{n}(\mathbb{Z})$ or & reference \\
& $\mathrm{GL}_{n}(\mathbb{Q})$ have order dividing & \\
\hline 1 & 2 & e.g. $[\mathrm{KP}, 4.1]$ \\
2 & $2^{3}$ or $2^{2} \cdot 3$ & {$[\mathrm{Ta}]$} \\
3 & $2^{4} \cdot 3$ & {$[\mathrm{Da}],[\mathrm{Ti} 92 \mathrm{~b}, \mathrm{p} .1137]$} \\
4 & $2^{7} \cdot 3^{2}$ or $2^{3} \cdot 5$ or $2^{4} \cdot 3 \cdot 5$ & \\
\hline
\end{tabular}

TABLE 3. Divisibility bound on finite subgroups of $\mathrm{GL}_{n}(\mathbb{Z})$ and $\mathrm{GL}_{n}(\mathbb{Q})$

The $F$-isomorphism class of an $n$-dimensional torus $T$ can be specified by a continuous homomorphism $\phi: \operatorname{Gal}(F) \rightarrow \mathrm{GL}_{n}(\mathbb{Z})$ (representing the natural Galois action on $T^{*}$ ), and $T$ is split over $F$ if and only if $\phi$ is trivial. It follows that every torus of rank $n$ can be split by a field extension of degree $|\operatorname{im} \phi|$, and this order is bounded as in Table 3 .

Let us start with the following elementary fact.

Proposition 3.1 ("type ${ }^{1} A_{p-1}$ "). Let $D$ be a division $F$-algebra of prime degree $p$ over a field $F$. Then the group $\mathrm{SL}_{1}(D)$ is almost abelian.

Proof. We follow 2.3. The group $\mathrm{SL}_{1}(D)$ is clearly anisotropic, and 2.3(2) holds because there are no proper semisimple subgroups of $\mathrm{SL}_{1}(D)$ of rank $p-1$ even over an algebraic closure of $F$.

It remains to show that every non-maximal torus in $\mathrm{SL}_{1}(D)$ is zero. First, we may replace $F$ by a co- $p$-closure $F_{p}$, because $D$ remains division over $F_{p}$. The torus $T$ is then a norm one torus $R_{K / F}^{1}\left(\mathbb{G}_{m}\right)$ for a cyclic Galois $F$-algebra $K$ of dimension $p$. Since $T$ is anisotropic, $K$ is a cyclic field extension of $F$. The corresponding representation $\operatorname{Gal}(K / F) \rightarrow$ $\mathrm{GL}\left(\mathbb{Z}^{p-1}\right) \rightarrow \mathrm{GL}\left(\mathbb{Q}^{p-1}\right)$ is $\mathbb{Q}$-irreducible. Thus $T$ does not admit nonzero proper subtori.

For contrast, we note that a group of type ${ }^{1} A_{n-1}$ where $n$ has at least two distinct prime factors is never almost abelian because a central simple algebra of degree $n$ is decomposable, see e.g. [GiS, 4.5.16].

Corollary 3.2 ("type ${ }^{2} A_{p-1}$ "). Let $p$ be an odd prime, and let $D$ be a central division algebra of degree $p$ over a quadratic extension $L / F$. If $D$ has a unitary involution $\tau$, then the group $\mathrm{SU}(D, \tau)$ is almost abelian.

Proof. Over $L$, the group $\mathrm{SU}(D, \tau)$ becomes isomorphic to $\mathrm{SL}_{1}(D)$, which is almost abelian by Prop. 3.1.

Before we go, we note the following:

Lemma 3.3. Every 3-dimensional torus over a 3-special field is isotropic. 
Proof. Over a 3 -special field $F$, a torus $T$ corresponds to a homomorphism $\phi: \operatorname{Gal}(F) \rightarrow \mathrm{GL}_{3}(\mathbb{Z})$ that necessarily factors through $\mathbb{Z} / 3 \mathbb{Z}$ by Table 3 . But any representation $\mathbb{Z} / 3 \mathbb{Z} \rightarrow \mathrm{GL}_{3}(\mathbb{Q})$ admits the trivial representation as direct summand, hence $T$ is isotropic.

\section{Global Fields}

We assume in this section that $F$ is a global field $F$.

Proposition 4.1. If an absolutely almost simple group $G$ over a global field $F$ is almost abelian, then $G$ has type type $A_{p-1}$ for some prime $p$.

Conversely, there exist almost abelian groups of type ${ }^{1} A_{n-1}$ and ${ }^{2} A_{n-1}$ over $F$ with $n$ prime by Prop. 3.1 and Cor. 3.2.

For the proof of Prop. 4.1, the only tricky case is that of outer type $A$. From Prasad-Rapinchuk's study of subalgebras of algebras with involutions [PrR 86, App. A], [PrR 08], we can derive the following fact.

Proposition 4.2. Let $L$ be a quadratic field extension of $F$. Let $A$ be a central simple L-algebra of degree $n$ equipped with an involution $\tau$ of the second kind such that $L^{\tau}=F$. If $\sqrt{-1} \in F$ or if $n$ is odd, there exists a cyclic field extension $E / F$ of degree $n$ such that $E \otimes_{F} L$ is a field and such that $\left(E \otimes_{F} L, \operatorname{Id} \otimes \tau\right)$ embeds in $(A, \tau)$.

Proof. Let $S$ be a finite set of places such that the group $\mathrm{SU}(A, \tau)$ is quasisplit for places away from $S$. We assume that $S$ contains a finite place $v_{0}$ such that $L \otimes_{F} F_{v}$ is not a field; such a place exists by Cebotarev density and if none are in $S$, then we add one.

For each place $v \in S$, we pick a cyclic étale $F_{v}$-algebra $E_{v}$ of degree $n$ such that $\left(E_{v}, \mathrm{Id}\right) \otimes_{F_{v}}\left(L \otimes F_{v}, \tau\right)$ embeds in $(A, \tau) \otimes_{F} F_{v}$ as an $\left(L \otimes_{F} F_{v}\right)$-algebra with involution.

Case 1: Suppose first that $L \otimes_{F} F_{v}$ is not a field. Then $A \otimes_{L}\left(L \otimes_{F} F_{v}\right) \cong$ $A_{v} \times A_{v}$ where $A_{v}$ is a central $F_{v}$-algebra.

If $v$ is finite, then by local class field theory, $A_{v}$ is split by the unramified extension of $F_{v}$ of degree $n$. We take it to be $E_{v}$.

If $v$ is infinite, then $F_{v}=\mathbb{C}$ or $n$ is odd. In either case, $A \otimes_{L}\left(L \otimes_{F} F_{v}\right)$ is split. Hence $\operatorname{SU}(A, \tau) \times{ }_{F} F_{v}$ is split; in this case we delete $v$ from $S$.

Case 2: Suppose now that $L \otimes_{F} F_{v}$ is a field $L_{w}$. We claim that the algebra $A \otimes_{L} L_{w}$ is split. It is obvious in the archimedean case; in the finite case, it follows from the bijectivity of the norm map $\operatorname{Br}\left(L_{w}\right) \rightarrow \operatorname{Br}\left(F_{v}\right)$. Then $(A, \tau) \otimes_{F} F_{v}$ is adjoint to a hermitian form for $L_{w} / F_{v}$. By diagonalizing it, it follows that $L_{w}^{n}$ embeds in $\left(A \otimes_{F} F_{v}, \tau\right)$. We take $E_{v}$ to be $F_{v}^{n}$.

The map

$$
H^{1}(F, \mathbb{Z} / n \mathbb{Z}) \rightarrow \prod_{v \in S} H^{1}\left(F_{v}, \mathbb{Z} / n \mathbb{Z}\right)
$$

is onto by [NSW, Cor. 9.2.3] (see also Ex. 1 on p. 459 of that book). So there exists a cyclic étale $F$-algebra $E$ such that $E \otimes F_{v}$ is isomorphic to $E_{v}$ 
for all $v \in S$. Note that $E$ is a field because $E_{v_{0}}$ is. Further, $L \otimes_{F} F_{v_{0}}$ is not a field, but $E \otimes_{F} F_{v_{0}}$ is, so $E$ does not contain a copy of $L$. As $E$ is Galois over $F$, it follows that $E \otimes L$ is a field.

By construction, for $v \in S$, the algebra with involution $\left(E_{v}, \mathrm{Id}\right) \otimes_{F_{v}}(L \otimes$ $\left.F_{v}, \tau\right)$ embeds in $(A, \tau) \otimes_{F} F_{v}$. But the same is true for $v$ not in $S$, because the group $\mathrm{SU}(A, \tau) \times F_{v}$ is quasi-split, cf. [PlR, p. 340]. By [PrR 08, Th. 3.1 $]^{2}$ (using that $E \otimes L$ is a field), $(E, \mathrm{Id}) \otimes(L, \tau)$ embeds in $(A, \tau)$ as $F$-algebras with involution.

Proof of Prop. 4.1. Let $G$ be an absolutely almost simple group over $F$, not of type $A_{n-1}$ for $n$ prime. We prove case-by-case that $G$ is not almost abelian.

Type ${ }^{1} A$ : If $G$ has type ${ }^{1} A_{n-1}$, then it is isogenous to $\operatorname{PGL}_{1}(A)$ where $A$ is a central simple algebra of degree $n$. By the Brauer-Hasse-Noether theorem, $A$ is a cyclic algebra, so contains a central simple proper subalgebra $A^{\prime}$ if $n$ is not prime. Then $\mathrm{PGL}_{1}\left(A^{\prime}\right)$ is a proper semisimple subgroup of $\mathrm{PGL}_{1}(A)$, hence $\mathrm{PGL}_{1}(A)$ is not almost abelian.

Type ${ }^{2} A$ : If $G$ has type ${ }^{2} A_{n-1}$ with $n$ not prime, it is isogenous to $\operatorname{SU}(B, \tau)$ where $B$ is a central simple algebra of degree $n \geq 3$ defined over a quadratic separable extension $L / F$ and equipped with an involution of the second kind. If $n$ is even, we know that $B$ contains a quadratic subalgebra $L^{\prime} / L$ stable under $\tau\left[\mathrm{K}, \S 5.7\right.$, p. 109]. So $\mathrm{SU}\left(L^{\prime}, \tau\right)$ is a rank one $k$-torus of $\mathrm{SU}(B, \tau)$, hence $\mathrm{SU}(B, \tau)$ is not almost abelian. If $n$ is odd, Proposition 4.2 shows that $(B, \tau)$ admits a subalgebra $\left(E \otimes_{F} L, \operatorname{Id} \otimes \tau\right)$ where $E$ is a cyclic Galois $F$-algebra of degree $n$. Because $n$ is not prime, there is a field $E_{0}$ such that $F \subsetneq E_{0} \subsetneq E$, and the special unitary group of $\left(E_{0} \otimes L, \operatorname{Id} \otimes \tau\right)$ is a non-maximal, non-zero torus in $\mathrm{SU}(B, \tau)$. Again $\mathrm{SU}(B, \tau)$ is not almost abelian.

Other types: Now suppose that $G$ is not of type $A$. If $F$ has no real places, we know that $G / F$ is isotropic (see [PIR, p. 352, Theorem 6.25] for the number field case). It is then not almost abelian. Further, inspecting the list of possible Tits indexes shows that in this case $G$ has a proper parabolic subgroup $P$ defined over $F$ that is reflexive but not a Borel.

If $F$ has real places, then we write $P$ for the parabolic subgroup of $G$ defined over $F(\sqrt{-1})$ from the previous sentence. It follows that $G$ has an $F$-subgroup $L$ that is conjugate over $F(\sqrt{-1})$ to a Levi subgroup of $P$ [PlR, p. 383, Lemma $6.17^{\prime}$ ], hence $G$ is not almost abelian.

\section{VERSAL GROUPS}

Here we define versal groups, which are the "most general" groups of a given quasi-split type.

\footnotetext{
${ }^{2}$ The January 2008 version of [PrR 08] includes the hypothesis that char $F \neq 2$, but it is not used in the proof of Theorem 3.1.
} 
Definition 5.1. Fix a field $F_{0}$, a connected Dynkin diagram $\Delta$, and an action of the absolute Galois group $\operatorname{Gal}\left(F_{0}\right)$ by graph automorphisms on $\Delta$, i.e., a continuous homomorphism $\phi: \operatorname{Gal}\left(F_{0}\right) \rightarrow \operatorname{Aut}(\Delta)$. There is a unique quasi-split simple adjoint group $G^{q}$ whose Tits index has underlying diagram $\Delta$ and Galois action $\phi$. Fix a versal $G^{q}$-torsor $V$ in the sense of [Se 03, §5]; it is a class $V \in H^{1}\left(F, G^{q}\right)$ for some finitely-generated, regular extension $F$ of $F_{0}$. Twisting $G^{q}$ by $V$ gives an inner form $G$ of $G^{q}$ defined over $F$.

A versal group is a group $G$ (over $F$ ) obtained in this manner from some $F_{0}, \Delta, \phi$. As an abbreviation, we say that $G$ is a versal group of type ${ }^{a} T_{n}$, for $T_{n}$ the Killing-Cartan type of $\Delta$ and $a=|\operatorname{im} \phi|$.

There is a further notion of versal groups when we allow $\phi$ to vary inside the morphisms $\phi: \operatorname{Gal}(F) \rightarrow \operatorname{Aut}(\Delta)$ with image in the conjugate in a subgroup $\Gamma \subset \operatorname{Aut}(\Delta)$.

Definition 5.2. Let $F_{0}$ and $\Delta$ be as above, and let $G^{d}$ be the split adjoint split group of type $\Delta$. Fix a subgroup $\Gamma \subset \operatorname{Aut}(\Delta)$. Write $\operatorname{Aut}_{\Gamma}\left(G^{d}\right)$ for the pull back by the inclusion $\Gamma \subset \operatorname{Aut}(\Delta)$ of the exact sequence

$$
1 \rightarrow G^{d} \rightarrow \operatorname{Aut}\left(G^{d}\right) \rightarrow \operatorname{Aut}(\Delta) \rightarrow 1
$$

Fix a versal $\operatorname{Aut}_{\Gamma}\left(G^{d}\right)$-torsor $V$ in the sense of $[\mathrm{Se} 03, \S 5]$; it is a class $V \in H^{1}\left(F, \operatorname{Aut}_{\Gamma}\left(G^{d}\right)\right)$ for some finitely-generated, regular extension $F$ of $F_{0}$. Twisting $G^{d}$ by $V$ gives a form $G$ of $G^{d}$ defined over $F$.

A superversal group is a group $G$ (over $F$ ) obtained in this manner from some $F_{0}, \Delta, \Gamma$. As an abbreviation, we say that $G$ is a superversal group of type ${ }^{a} T_{n}$, for $T_{n}$ the Killing-Cartan type of $\Delta$ and $a=|\Gamma|$.

The case $\Gamma=1$, is nothing but the versal inner form.

5.3. Maintain the notation of Definition 5.2. Suppose that $M$ is an adjoint group with the same Dynkin diagram $\Delta$, defined over an extension $E$ of $F_{0}$, and the *-action on the Dynkin diagram of $M$ belongs to the image of $H^{1}(E, \Gamma) \rightarrow H^{1}(E$, Aut $(\Delta))$. We claim that $M$ is in the image of the map $H^{1}\left(E, \operatorname{Aut}_{\Gamma}\left(G^{d}\right)\right) \rightarrow H^{1}\left(E, \operatorname{Aut}\left(G^{d}\right)\right)$.

We have to check that this kind of groups occur as twisted forms of $G^{d}$ by cocycles with value in $\operatorname{Aut}_{\Gamma}\left(G^{d}\right)$. There exists $[z] \in H^{1}\left(E, \operatorname{Aut}\left(G^{d}\right)\right)$ such that $M \cong{ }_{z} G^{d}$. We consider the commutative diagram

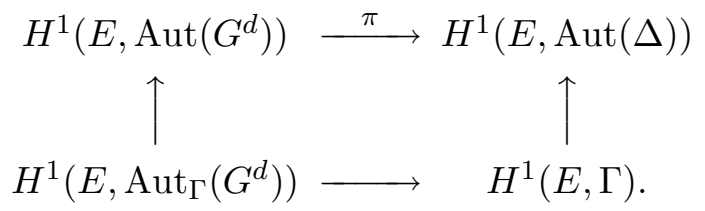

Our hypothesis is that $\pi_{*}[z] \in \operatorname{Im}\left(H^{1}(E, \Gamma) \rightarrow H^{1}(E\right.$, Aut $\left.(\Delta))\right)$. According to [Se 02, §I.5, prop. 37], this is equivalent to the fact that $\pi_{*}(z)(\operatorname{Aut}(\Delta) / \Gamma)(E) \neq$ $\emptyset$. This finite set is nothing but $\pi_{*}(z)\left(\operatorname{Aut}\left(G^{d}\right) / \operatorname{Aut}_{\Gamma}\left(G^{d}\right)\right)(E)$ hence the claim. 
We conclude this section by the following observation.

Lemma 5.4. Let $\Psi$ be a root datum in the sense of Springer [Sp, 7.4.1]. The following are equivalent:

(1) The superversal group $G / F$ contains a reductive subgroup of type $\Psi$.

(2) For every field extension $E / F_{0}$ and every adjoint group $M / E$ of type $\Delta$ such that $\varphi_{M} \in \operatorname{Im}\left(H^{1}(E, \Gamma) \rightarrow H^{1}(E, \operatorname{Aut}(\Delta))\right)$, the group $M / E$ has a reductive subgroup of type $\Psi$.

Note that there is an analogous statement for versal groups.

We use a root datum instead of a root system so as to include also tori and the kernel of the map from the simply connected cover.

Proof. (2) $\Longrightarrow(1)$ is obvious. Conversely, assume that $G / F$ admits a $F$ subgroup $H \subset G / F$ of type $\Psi$. The $\operatorname{Aut}_{\Gamma}(G)$-torsor $V / F$ arises as the generic fiber of a $\operatorname{Aut}_{\Gamma}(G)$-torsor $\mathfrak{V} / X$ where $X$ is a smooth $F_{0}$-variety such that $F=F_{0}(X)[$ Se 03, $\S 5]$. Define $\mathfrak{G} / X$ as the twisted $X$-form of $G^{d}$ by $\mathfrak{V}$. The idea is to extend $H$ locally. The quotient $Q=G / H$ is a $G$-variety over $F$. According to $\left[\mathrm{SGA} 3, \mathrm{VI}_{B} \cdot 10.16\right]$ there exists an open (non empty) Zariski-connected subset $U \subset X$ and a homogeneous $\mathfrak{G}$-scheme $\mathfrak{Q} / U$ such that $Q=\mathfrak{Q} \times_{U} F_{0}(X)$. Shrinking $U$ further if necessary, we can assume that the origin of $Q$ extends to a point $q \in \mathfrak{Q}(U)$. We define the closed $U$-subgroup scheme $\mathfrak{H}:=\operatorname{Stab}_{\mathfrak{G}}(q)$. Again, shrinking $U$ if necessary, we can assume that $\mathfrak{H} / U$ is smooth and connected. By [SGA3, XIX.2.6], $\mathfrak{H} / U$ is a reductive group scheme. By Demazure's type unicity theorem [SGA3, XXII.2.8], $\mathfrak{H}$ is of type $\Psi$, i.e., all geometric fibers are of type $\Psi$.

We can now proceed to the proof. We are given a field $E / F_{0}$ and an adjoint group $M / E$ of type $\Delta$ such that its $*$-action $\varphi_{M} \in H^{1}(E, \operatorname{Aut}(\Delta))$ comes from $H^{1}(F, \Gamma)$. By 5.3 , there exists $x \in U(E)$ such that $M$ is isomorphic to the fiber $x^{*} \mathfrak{G}$. Then $M / E$ admits as the closed subgroup $x^{*} \mathfrak{H}$ which is reductive of type $\Psi$.

Corollary 5.5. Let $G$ be a superversal group as in Definition 5.2, and assume that the characteristic of $F_{0}$ is not a torsion prime for $G$. The following are equivalent:

(1) The superversal group $G / F$ is not almost abelian.

(2) For every field extension $E / F_{0}$ and every adjoint group $M / E$ of type $\Delta$ such that $\varphi_{M} \in \operatorname{Im}\left(H^{1}(E, \Gamma) \rightarrow H^{1}(E, \operatorname{Aut}(\Delta))\right)$, the group $M / E$ is not almost abelian.

Proof. Again $(2) \Longrightarrow(1)$ is obvious. Conversely, assume that $G / F$ admits a proper non zero $F$-subgroup $H \subset G$. Since $G$ is anisotropic, $H$ is reductive. Hence Lemma 5.4 applies. 


\section{ROST INVARIANT AND ADJOINT GROUPS}

6.1. Let $G^{q}$ be a quasi-split simple adjoint algebraic group, and write $\widetilde{G}^{q}$ for its simply connected cover. The Rost invariant of $\widetilde{G}^{q}$ is a morphism of functors Fields $_{/ F} \rightarrow$ Sets:

$$
r_{\widetilde{G}^{q}}: H^{1}\left(*, \widetilde{G}^{q}\right) \rightarrow H^{3}(*, \mathbb{Z} / \delta \mathbb{Z}(2)),
$$

where $\delta$ is the Dynkin index of $\widetilde{G}^{q}$ as defined in [Mer 03, §10]. (We remind that the group $H^{3}(F, \mathbb{Z} / d \mathbb{Z}(2))$ has an unusual definition [Mer 03, App. A]. In case the characteristic of $F$ does not divide $d$, it is the Galois cohomology group $H^{3}\left(F, \mu_{d}^{\otimes 2}\right)$.) Factor $\delta$ as $\delta_{0} m$, where $m$ is maximal with the property of being relatively prime to both $\delta_{0}$ and the exponent of the group $Z^{*}\left(F_{\text {sep }}\right)$

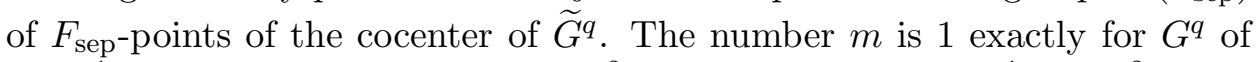
type ${ }^{1} A_{n}, B_{n}$, or $C_{n}$ for all $n$; type ${ }^{2} A_{n}$ for $n$ odd; or type ${ }^{1} D_{n}$ or ${ }^{2} D_{n}$ for $n \geq 4$. The number $m$ is $\delta$ when $Z$ is trivial, i.e., for $G^{q}$ of type $E_{8}, F_{4}$, or $G_{2}$. All the other cases -i.e., where $m \neq 1, \delta$ - are given in Table 6 .

\begin{tabular}{l|ccc|}
\hline type of $G^{q}$ & $m$ & Dynkin index $\delta$ of $\widetilde{G}^{q}$ & exponent of $Z^{*}\left(F_{\text {sep }}\right)$ \\
\hline${ }^{2} A_{n}(n$ even $)$ & 2 & 2 & $n+1$ \\
${ }^{3} D_{4},{ }^{6} D_{4}$ & 3 & 6 & 2 \\
${ }^{1} E_{6}$ & 2 & 6 & 3 \\
${ }^{2} E_{6}$ & 4 & 12 & 3 \\
$E_{7}$ & 3 & 12 & 2 \\
\hline TABLE & 6. Factorizations of Dynkin indexes for simple \\
groups. All other quasi-split simple groups have $m=1$ or \\
$m=\delta$.
\end{tabular}

For each extension $K / F$, the group $H^{3}(K, \mathbb{Z} / \delta \mathbb{Z}(2))$ can naturally be written as a direct sum of its $m$ and $\delta_{0}$ torsion parts, i.e., as $H^{3}(K, \mathbb{Z} / m \mathbb{Z}(2)) \oplus$ $H^{3}\left(K, \mathbb{Z} / \delta_{0} \mathbb{Z}(2)\right)$.

Proposition 6.2. Suppose the characteristic of $F$ does not divide $m$. Then there is a unique invariant

$$
r_{G^{q}, m}: H^{1}\left(*, G^{q}\right) \rightarrow H^{3}(*, \mathbb{Z} / m \mathbb{Z}(2))
$$

such that the diagram

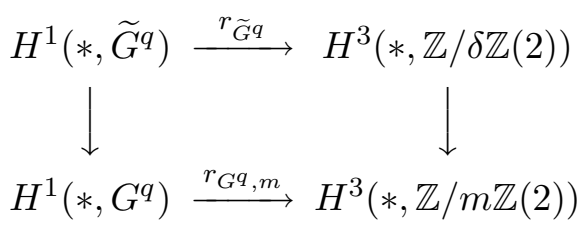

commutes. 
Proof. The result is trivial if $\widetilde{G}^{q}=G^{q}$ (i.e., $m=\delta$ ) or $m=1$, so we may assume that $m$ is given by Table 6 . In particular, $m$ is a power of some prime $p$.

Fix an element $y \in H^{1}(K, G)$ for some extension $K / F$; we claim that there is an extension $L / K$ such that $\operatorname{res}_{L / K}(y)$ is in the image of $H^{1}\left(L, \widetilde{G}^{q}\right) \rightarrow$ $H^{1}(L, G)$ and $[L: K]$ is relatively prime to $m$. Let $K_{p}$ denote the co- $p$ closure of the perfect closure of $K$. The group $H_{\text {fppf }}^{2}\left(K_{p}, Z\right)$ is zero because the exponent of $Z$ is not divisible by $p$. The exactness of the sequence $1 \rightarrow Z \rightarrow \widetilde{G}^{q} \rightarrow G^{q} \rightarrow 1$ implies the existence of $L$.

Now suppose that $x_{1}, x_{2} \in H^{1}\left(L, \widetilde{G}^{q}\right)$ both map to $y$. Then there is a $z \in H_{\mathrm{fppf}}^{1}(L, Z)$ such that $z \cdot x_{1}=x_{2}$. Applying the Rost invariant, we find

$$
r_{\widetilde{G}^{q}}\left(x_{1}\right)=r_{\widetilde{G}^{q}}\left(z \cdot x_{2}\right)=r_{\widetilde{G}^{q}}\left(x_{2}\right)
$$

by [Gi, p. 76, Lemma 7], cf. [GaQ, Remark 2.5(i)]. Combining this with the previous paragraph proves the existence and uniqueness of $r_{G^{q}, m}$ by [Ga, Prop. 7.1] (which uses the hypothesis on the characteristic of $F$ ).

Definition 6.3. Let $G$ be a simple algebraic group over $F$, and write $\bar{G}$ for its associated adjoint group. There is a unique class $\eta \in H^{1}(F, \bar{G})$ such that the group $\bar{G}_{\eta}$ obtained by twisting $\bar{G}$ by $\eta$ is quasi-split [KMRT, 31.6]. Put $m$ for the natural number defined for the quasi-split group $\bar{G}_{\eta}$ in 6.1, and suppose that the following holds:

The characteristic of $F$ does not divide $m$ or $G$ has type $F_{4}, G_{2}$, or $E_{8}$.

Write $\theta_{\eta}$ for the twisting isomorphism $H^{1}\left(F, \bar{G}_{\eta}\right) \stackrel{\sim}{\rightarrow} H^{1}(F, \bar{G})$ and define:

$$
r(G):=r_{\bar{G}_{\eta}, m}\left(\theta_{\eta}^{-1}(0)\right) \quad \in H^{3}(F, \mathbb{Z} / m \mathbb{Z}(2)) \subseteq H^{3}(F, \mathbb{Q} / \mathbb{Z}(2)) .
$$

This element depends only on the isomorphism class of $G$. If $G$ is one of the groups listed in Table 6, then $m$ divides 24 and $r(G)$ takes values in $H^{3}(F, \mathbb{Z} / m \mathbb{Z})[\mathrm{KMRT}, \S \mathrm{VII}$, exercise 11].

It is easy to see that $r$ is an invariant in the sense of [Se 03], e.g., for every extension $K / F$ we have:

$$
\operatorname{res}_{K / F}(r(G))=r\left(G \times_{F} K\right) .
$$

Lemma 6.5. If $G$ is a versal group and (6.4) holds, then $r(G)$ has order $m$.

Proof. The claim is equivalent to the following statement in the language of Prop. 6.2: If $V$ is a versal $G^{q}$-torsor, then $r_{G^{q}, m}(V)$ has order $m$ in $H^{3}(F, \mathbb{Z} / m \mathbb{Z}(2))$. To prove this, by the specialization property of versal torsors [Se 03, 12.3], it suffices to produce an element $y \in H^{1}\left(E, G^{q}\right)$ for some extension $E / F$ such that $r_{G^{q}, m}(y)$ has order $m$ in $H^{3}(E, \mathbb{Z} / m \mathbb{Z}(2))$. We take $y$ to be the image of a versal $\widetilde{G}^{q}$-torsor $\tilde{V}$. By [Mer 03, 10.8], the element $r_{\widetilde{G}^{q}}(\tilde{V})$ has order $\delta$, and the claim follows. 


\section{Groups of type $E_{8}$}

Besides being split or anisotropic, the possible indexes for a group $G$ of type $E_{8}$ are given by Table 7 .

\begin{tabular}{|c|c|}
\hline $\begin{array}{c}\text { semisimple anisotropic } \\
\text { kernel of } G\end{array}$ & order of $r(G)$ \\
\hline$E_{7}$ & $\neq 1$, divides 12 \\
$D_{7}$ & 2 \\
$E_{6}$ & 3 or 6 \\
$D_{6}$ & 2 \\
$D_{4}$ & 2 \\
\hline
\end{tabular}

TABle 7. Possible invariants of a group $G$ of type $E_{7}$ that is neither split nor anisotropic.

Lemma 7.1 (Cf. [Ti 91, p. 134, Prop. 5]). Let $G$ be a group of type $E_{8}$ over a 5-special field $F$. If $G$ is not split, then $G$ is anisotropic and every proper, nonzero reductive subgroup of $G$ is a torus of rank 4 or 8 or has semisimple type $A_{4}$ or $A_{4} \times A_{4}$.

We note that all of these possibilities can occur, see [Ga, 15.7].

Proof. As $G$ is not split, if it is isotropic, then $r(G)$ is not zero by Table 7. But $G$ is split by a separable extension of $F$, so $r(G)$ has order dividing 5. It follows from Table 7 that $G$ is anisotropic. This implies by Table 3 that $G$ cannot contain tori of ranks 1, 2, or 3. By the previous sentence, every nonzero reductive subgroup of $G$ has rank 4 or 8 . It is easy to check that any semisimple group of rank 4 over $F$ is isotropic or of type $A_{4}$. For a subgroup of rank 8, we consult the list of such in [Dy, Table 10]; in addition to $A_{4} \times A_{4}$, we find:

(1) $A_{7} \times A_{1}, A_{5} \times A_{2} \times A_{1}, E_{6} \times A_{2}, E_{7} \times A_{1}, D_{6} \times A_{1}^{\times 2}, D_{5} \times A_{3}, A_{3}^{\times 2} \times A_{1}^{\times 2}$

(2) $A_{8}, D_{8}, A_{2}^{\times 4}, D_{4}^{\times 2}, D_{4} \times A_{1}^{\times 4}, A_{1}^{\times 8}$

Those in list (1) do not occur because they contain tori of ranks $\neq 4,8$. Those in list (2) do not occur because they are isotropic over a 5 -special field.

Lemma 7.2. Let $G$ be a non-split group of type $E_{8}$ over a 3 -special field.

(1) If $G$ is isotropic, it has rank 2 and semisimple anisotropic kernel of type $E_{6}$.

(2) $G$ does not contain a semisimple subgroup of type $A_{4}$ or $A_{4} \times A_{4}$.

Proof. Over a 3-special field, every group of type $E_{7}$ is isotropic [Ga, 13.1], so (1) follows from Table 7. Since the field is 3-special, a semisimple group of type $A_{4}$ or $A_{4} \times A_{4}$ is split, contradicting the statement that $G$ has rank at most 2 . 
Theorem 7.3. In a versal group of type $E_{8}$, every nonzero proper reductive subgroup is a rank 8 torus.

Proof. Every group of type $E_{8}$ is simply connected, so the versal group $G$ is obtained by twisting the split group $G^{q}$ of type $E_{8}$ by a versal torsor. By [Mer 03, 16.8], the Rost invariant $r_{G^{q}}(G)$ has order 60 in $H^{3}(F, \mathbb{Z} / 60 \mathbb{Z}(2))$. In particular, this element is not killed by a co-3-closure nor a co-5-closure of $F$ and so $G$ is not split over such fields. Combining Lemmas 7.1 and 7.2, it suffices to show that $G$ does not contain any rank 4 tori.

For sake of contradiction, suppose that $G$ has a rank 4 torus $S$ defined over $F$, and that an extension $L / F$ splits $S$. Then $G$ has $L$-rank at least 4 , and so $G$ is $L$-split or has semisimple anisotropic kernel a strongly inner form of $D_{4}$ over $L$; in either case $G$ is split by an extension of $F$ of degree dividing $2[L: F]$. By [To], the torsion index of the compact real Lie group $E_{8}$ is 2880 , hence every extension that splits $G$ has dimension divisible by 2880 , hence $[L: F]$ is divisible by $1440=2^{5} \cdot 3^{2} \cdot 5$. But by Table 3, every rank 4 torus over $F$ is split by an extension of degree dividing $2^{7} \cdot 3^{2}$ or $2^{3} \cdot 5$ or $2^{4} \cdot 3 \cdot 5$. This is a contradiction.

Applying 2.5 gives:

Corollary 7.4 (Cf. [Ti 92b, p. 1135, Cor. 3]). Every versal group $G$ of type $E_{8}$ over a field of characteristic $\neq 2,3,5$ is almost abelian.

In the proof of Th. 7.3, the second paragraph amounts to a reference to Totaro's general result on the torsion index; this has the advantage of being very easy. Alternatively, one could replace it with a reference to [Ti $92 \mathrm{~b}$, Lemme 5], which says: If a group $G$ of type $E_{8}$ contains a regular rank 4 torus, then $G$ is split by an extension of dimension dividing $2^{7} \cdot 3^{2}$ or $2^{3} \cdot 5$. This gives a strengthening of Th. 7.3:

Theorem 7.5. If $G$ is a group of type $E_{8}$ such that $r(G)$ has order 60 , then every nonzero proper reductive subgroup of $G$ is a maximal torus.

\section{Tits Algebras}

Let $G$ be a semisimple algebraic group over $F$. Tits defined certain invariants $\beta_{G}(\chi)$ of $G$ in [Ti 71] with the following properties, cf. [KMRT, §27] and [Mer 96, §4]. Write $Z$ for the center of a simply connected cover of $G$. The Cartier dual $Z^{*}$ of the center - the cocenter - is an étale group scheme. For $\chi \in Z^{*}\left(F_{\mathrm{sep}}\right)$, we put $F_{\chi}$ for the subfield of $F_{\text {sep }}$ fixed by the stabilizer of $\chi$ in $\operatorname{Gal}(F)$. The Tits algebra $\beta_{G}(\chi)$ is an element of $H^{2}\left(F_{\chi}, \mathbb{G}_{m}\right)$, the Brauer group of $F_{\chi}$.

Let $t_{G} \in H_{\text {fppf }}^{2}(F, Z)$ be the Tits class of $G$ [KMRT, $\left.\S 31\right]$. We note the following properties:

(8.1) For all $\chi \in Z^{*}\left(F_{\mathrm{sep}}\right)$, the image of $t_{G}$ under the composite map

$$
H_{\mathrm{fppf}}^{2}(F, Z) \stackrel{\text { res }}{\longrightarrow} H_{\mathrm{fppf}}^{2}\left(F_{\chi}, Z\right) \stackrel{\chi_{*}}{\longrightarrow} H^{2}\left(F_{\chi}, \mathbb{G}_{m}\right)
$$


is $\beta_{G}(\chi)$, see [KMRT, 31.7].

(8.2) For $G$ of inner type, the Galois group acts trivially on $Z^{*}\left(F_{\mathrm{sep}}\right)$ and $F_{\chi}=F$ for all $\chi$.

(8.3) The Galois action on $Z^{*}$ is "compatible" with the Tits algebras in the following sense: For $\sigma \in \operatorname{Gal}(F)$, clearly $F_{\sigma \chi}$ equals $\sigma\left(F_{\chi}\right)$. Further, the natural map

$$
\sigma: H^{2}\left(F_{\chi}, \mathbb{G}_{m}\right) \rightarrow H^{2}\left(F_{\sigma \chi}, \mathbb{G}_{m}\right)
$$

sends $\beta_{G}(\chi)$ to $\beta_{G}(\sigma \chi)$. This follows from (8.1). In particular, the index of the division algebra representing $\beta_{G}(\chi)$ is the same as the index of the algebra representing $\beta_{G}(\sigma \chi)$.

(8.4) The Tits algebras are compatible with scalar extension in a natural way. If $K / F$ is an extension contained in $F_{\text {sep }}$, then $K_{\chi}$ is the compositum of $F_{\chi}$ and $K$, and $\beta_{G \times K}(\chi)$ is the restriction $\operatorname{res}_{K_{\chi} / F_{\chi}} \beta_{G}(\chi)$.

(8.5) For every integer $k$, the element $\operatorname{res}_{F_{\chi} / F_{k \chi}} \beta_{G}(k \chi)$ equals $k \cdot \beta_{G}(\chi)$ in $H^{2}\left(F_{\chi}, \mathbb{G}_{m}\right)$, by $(8.1)$.

There is a number $n_{G}(\chi)$ - depending only on $\chi$ and the absolute KillingCartan type of $G$-such that the index of a division algebra representing $\beta_{G}(\chi)$ divides $n_{G}(\chi)$, cf. [Ti $71,5.4$. It is defined as follows: If $\chi$ is zero then $n_{G}(\chi)$ is defined to be 1 . Otherwise, there is a unique Weyl orbit of minuscule weights whose restriction to $Z$ is $\chi$; write $c$ for the cardinality of the orbit. The number $n_{G}(\chi)$ is the largest divisor of $c$ that is only divisible by primes dividing the order of $Z^{*}\left(F_{\text {sep }}\right)$. The numbers $n_{G}(\chi)$ are computed in [Ti 71, §6], [MPW1], and [MPW2].

Property (8.5) immediately implies:

(8.6) If $k$ is relatively prime to the exponent of $Z^{*}\left(F_{\mathrm{sep}}\right)$, then $\beta_{G}(k \chi)$ and $\beta_{G}(\chi)$ have the same index. Indeed, $\chi$ and $k \chi$ generate the same subgroup of $Z^{*}\left(F_{\text {sep }}\right)$, hence $F_{k \chi}$ equals $F_{\chi}$. The claim amounts to a standard fact about elements of the Brauer group of $F_{\chi}$ : The index of a class $\alpha$ is equal to the dimension of the smallest separable extension of $F_{\chi}$ that kills $\alpha$, equivalently the subgroup generated by $\alpha$.

About the Tits algebras of versal/superversal groups, our main tool is the following theorem of Merkurjev:

Theorem 8.7 (Th. 5.2 of [Mer 96]). If $G$ is a versal group and $\chi$ is fixed by $\operatorname{Gal}(F)$, then the index of the Tits algebra $\beta_{G}(\chi)$ is $n_{G}(\chi)$.

Alternatively, one can find concrete constructions for $G$ of inner type in [MPW1] and [MPW2]. We extend this theorem in $\S 12$ below.

\section{WEYL GROUP OF $E_{6}$}

This section prepares the ground for the proof of Prop. 10.1. 
9.1. We label the simple roots in a root system of type $E_{6}$ as in the extended Dynkin diagram

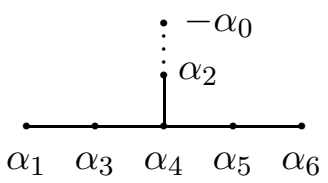

where $\alpha_{0}$ denotes the highest root. The roots

$$
\alpha_{1}, \alpha_{3}, \alpha_{5}, \alpha_{6}, \alpha_{2},-\alpha_{0}
$$

are a basis for a root subsystem of type $A_{2} \times A_{2} \times A_{2}$, and we refer to it simply as $A_{2}^{\times 3}$. Of course, all subsystems of $E_{6}$ of that type are conjugate under the Weyl group $W\left(E_{6}\right)$ [Bou, Exercise VI.4.4].

Lemma 9.3. There is exactly one conjugacy class of elements of $W\left(E_{6}\right)$ of order 2 , determinant 1 , and trace -2 . Every element of the class normalizes a sub-root-system of type $A_{2}^{\times 3}$.

Here, the determinant and trace are computed relative to the action of $W\left(E_{6}\right)$ on the $\mathbb{Q}$-vector space spanned by the roots.

Proof of Lemma 9.3. The determinant defines an exact sequence

$$
1 \longrightarrow \Gamma \longrightarrow W\left(E_{6}\right) \stackrel{\operatorname{det}}{\longrightarrow} \pm 1 \longrightarrow 1
$$

where $\Gamma$ is the finite simple group $U_{4}(2)$, cf. [Bou, Exercise VI.4.2]. To prove the first claim, it suffices to note that there is exactly one conjugacy class of elements of $\Gamma$ of order 2 and trace -2 , as can be read off of the 4 th line of the table on p. 27 of the Atlas [Atlas].

For the second claim, take $w$ to be the composition of the reflections in the (pairwise orthogonal) roots

$$
\begin{array}{cc}
\alpha_{1}+\alpha_{3}+\alpha_{4}+\alpha_{5} & \alpha_{2}+\alpha_{3}+\alpha_{4}+\alpha_{5} \\
\alpha_{5}+\alpha_{6} & \alpha_{1}+\alpha_{2}+2 \alpha_{3}+2 \alpha_{4}+\alpha_{5}+\alpha_{6}
\end{array}
$$

Clearly, $w$ has determinant 1 . It interchanges $\alpha_{1}, \alpha_{3}$ with $\alpha_{2},-\alpha_{0}$ respectively and sends $\alpha_{5}, \alpha_{6}$ to $-\alpha_{5},-\alpha_{6}$. It follows that $w$ has trace -2 and stabilizes the subsystem $A_{2}^{\times 3}$.

Example 9.4. The roots $\alpha_{2}, \alpha_{3}, \alpha_{4}, \alpha_{5}$ span a sub-root-system of type $D_{4}$ in $E_{6}$, and this gives an inclusion $W\left(D_{4}\right) \hookrightarrow W\left(E_{6}\right)$. The element -1 of $W\left(D_{4}\right)$ maps to an element $w \in W\left(E_{6}\right)$ of order 2. This element acts as -1 on the 4-dimensional subspace spanned by the roots in $D_{4}$ and fixes the (orthogonal) 2-dimensional subspace spanned by the fundamental weights of $E_{6}$ dual to $\alpha_{1}$ and $\alpha_{6}$. It follows that $w$ has determinant 1 and trace -2 . 


\section{Groups of Type ${ }^{1} E_{6}$}

Let $G$ be a group of type ${ }^{1} E_{6}$. The cocenter $Z^{*}$ is isomorphic to $\mathbb{Z} / 3 \mathbb{Z}$. The numbers $n_{G}(\chi)$ are 27 for nonzero $\chi$, and the Tits algebras $\beta_{G}(1)$ and $\beta_{G}(2)$ have the same index by (8.6). We prove:

Proposition 10.1. Let $G$ be a group of type ${ }^{1} E_{6}$ over a field $F$ of characteristic $\neq 2,3$.

(1) If $F$ is a p-special field for some prime $p$, then $G$ contains a subgroup of type $A_{2}^{\times 3}$.

(2) [Ti 91, p. 129] If $\beta_{G}(1)$ has index 27, then $G$ has no nonzero, proper, closed connected $F$-subgroups other than maximal tori and possibly subgroups of semisimple type $A_{2}^{\times 3}$.

Versal groups have $\beta_{G}(1)$ of index 27 , so (2) is not empty. (Alternatively, an explicit construction of a group of type ${ }^{1} E_{6}$ as in (2) and containing a semisimple subgroup of type $A_{2}^{\times 3}$ can be found in [MPW2, pp. 153-155].) We note that the statement of Prop. 10.1(2) applies equally well to groups of type ${ }^{2} E_{6}$, and that there exist such groups $G$ with $\beta_{G}(1)$ of index 27 by Th. 12.1(2) below.

Besides being split or anisotropic, the possible indexes for $G$ are given by Table 10.

\begin{tabular}{|c|cc|}
\hline $\begin{array}{l}\text { semisimple anisotropic } \\
\text { kernel of } G\end{array}$ & index of $\beta_{G}(1)$ & $r(G)$ \\
\hline$A_{2} \times A_{2}$ & 3 & 0 \\
$D_{4}$ & 1 & $\neq 0$ \\
\hline
\end{tabular}

TABle 10. Possible invariants of a group $G$ of type ${ }^{1} E_{6}$ that is neither split nor anisotropic.

Remark 10.2. We take this opportunity to fill a tiny gap from the classification of possible Tits indexes on p. 311 of [Sp]. That reference omits a proof that a group $G$ of type ${ }^{1} E_{6}$ over a field of characteristic 3 cannot have semisimple anisotropic kernel of type $A_{5}$. To see this, suppose that $G$ has such a semisimple anisotropic kernel; call it $M$. It is isomorphic to $\mathrm{SL}_{1}(D)$ for a division algebra $D$ of index 6 . The fundamental weight $\omega_{4}$ corresponding to the simple root $\alpha_{4}$ of $G$ belongs to the root lattice of $E_{6}$, so the Tits algebra $\beta_{G}\left(\omega_{4}\right)$ is zero. On the other hand, the restriction of $\omega_{4}$ to $M$ has Tits algebra the Brauer class of $D \otimes D \otimes D$. By [Ti71, p. 211], these two Brauer classes are equal, so $D^{\otimes 3}$ is split and $M$ is isotropic, a contradiction.

Proof of Prop. 10.1(2). It suffices to prove this in the case where $F$ is 3special. By Table 10, $G$ is anisotropic, and remains anisotropic over every cubic extension of $F$. It follows from Table 3 that $G$ cannot contain $F$-tori of dimensions 1,2 , or 3 , nor dimensions 4 or 5 by symmetry. Consulting 
[Dy, Table 10], the only possible types for proper semisimple subgroups of maximal rank are $A_{5} \times A_{1}$ and $A_{2}^{\times 3}$. But the first type contains a 1dimensional torus, so it cannot be the type of an $F$-subgroup of $G$.

Lemma 10.3. Let $G$ be a group of type ${ }^{1} E_{6}$ over a field $F$ of characteristic $\neq 2$. If $G$ is split by an extension of $F$ of degree not divisible by 3 , then $G$ contains a subgroup of type $A_{2}^{\times 3}$.

Proof. The hypothesis on $G$ implies that $G$ is split-in which case we are done - or $G$ is isotropic with semisimple anisotropic kernel of type $D_{4}$.

Write $E_{6}, D_{4}, G_{2}$ for the split simply connected groups of those types. We view $D_{4}$ as a subgroup of $E_{6}$ via the inclusion from Example 9.4 and $G_{2}$ as the subgroup of $D_{4}$ consisting of elements fixed by the outer automorphism $\phi$ of order 3 that cyclically permutes the simple roots $\alpha_{2}, \alpha_{3}, \alpha_{5}$. (Recall that the roots of $E_{6}$ are all relative to some fixed split maximal torus $T$.)

Write $n$ for an element of $D_{4}$ normalizing $T$ and representing -1 in the Weyl group of $D_{4}$. Replacing $n$ with $n \phi(n) \phi^{2}(n)$, we may assume that $n$ belongs to $G_{2}$. Write $A$ for the group generated by $n$ and a maximal torus $T_{2}:=\left(T \cap G_{2}\right)^{0}$ in $G_{2}$. The natural map $H^{1}(F, A) \rightarrow H^{1}\left(F, G_{2}\right)$ is surjective, cf. [CS, p. 1060]. Further, the image of $H^{1}\left(F, G_{2}\right) \rightarrow H^{1}\left(F, \operatorname{Aut}\left(D_{4}\right)\right)$ contains the class of the semisimple anisotropic kernel of $G$, because 8dimensional quadratic forms in $I^{3}$ are similar to Pfister forms. Combining these two observations with Tits's Witt-type theorem [Sp, 16.4.2], we deduce that the simply connected cover of $G$ is obtained by twisting $E_{6}$ by a 1-cocycle $\eta$ with values in $A$. By Lemma 9.3, $\eta$ stabilizes a sub-root-system of type $A_{2}^{\times 3}$, hence the claim.

Proof of Prop. 10.1(1). Suppose that $p=3$; otherwise the claim is Lemma 10.3. The adjoint quotient of $G$ is obtained by twisting the split adjoint $E_{6}$ by a 1-cocycle $\eta$ with values in the normalizer $N$ of a maximal split torus $T$ such that the image of $\eta$ in $N / T$ is contained in a 3-Sylow subgroup of $W\left(E_{6}\right)$ of our choosing. It suffices to note that one can find a 3-Sylow in $W\left(E_{6}\right)$ that normalizes the subsystem $A_{2}^{\times 3}$ from 9.1, cf. [C, Lemma 5.8].

\section{Groups of TYPE $E_{7}$}

In this section we consider groups $G$ of type $E_{7}$. The cocenter of the simply connected cover of $G$ is $\mathbb{Z} / 2 \mathbb{Z}$, and the Tits algebra $\beta_{G}(1)$ has index dividing $n_{G}(1)=8$. The element $r(G)$ belongs to $H^{3}(F, \mathbb{Z} / 3 \mathbb{Z})$. Our goal is to prove the following:

Proposition 11.1. Let $G$ be a group of type $E_{7}$ over a field $F$ of characteristic zero.

(1) If $F$ is p-special, then $G$ contains semisimple subgroups of type $D_{4}, A_{1}^{\times 3}$, and $D_{4} \times A_{1}^{\times 3}$.

(2) If $\beta_{G}(1)$ has index 8 and $r(G)$ is nonzero, then every proper semisimple subgroup of $G$ (if there are any) is normalized by a maximal torus of $G$ and has type $D_{4}, A_{1}^{\times 3}$, or $D_{4} \times A_{1}^{\times 3}$. 
We remark that groups of type $D_{4}$ and $A_{1}^{\times 3}$ can be almost abelian by Theorem 1.1 and Example 2.6. Also, groups $G$ as in (2) exist; for example, a versal group satisfies the hypothesis by Lemmas 8.7 and 6.5 .

Proof of Prop. 11.1(1). If the group of type $E_{7}$ is split (e.g., if $p \neq 2,3$ ), then the result is clear.

If $F$ is 2-special, we note that the Weyl group of $E_{7}$ and of the standard subgroup of type $D_{6} \times A_{1}$ have the same 2 -Sylows, so every $F$-group of type $E_{7}$ has an $F$-subgroup of type $D_{6} \times A_{1}$, cf. e.g. [Ga, 14.7]. Then it suffices to note that every group of type $D_{6}$ contains a subgroup of type $D_{4} \times D_{2}$, i.e., $D_{4} \times A_{1}^{\times 2}$.

If $F$ is 3 -special, then every simply connected group of type $E_{7}$ is obtained by twisting the split group by a 1-cocycle that normalizes the standard $D_{4}$ subgroup [Ga, 13.1]. The centralizer of this $D_{4}$ subgroup has type $A_{1}^{\times 3}$, corresponding to the sub-root-system spanned by

$$
\begin{gathered}
\alpha_{7}, \quad \alpha_{2}+\alpha_{3}+2 \alpha_{4}+2 \alpha_{5}+2 \alpha_{6}+\alpha_{7}, \quad \text { and } \\
2 \alpha_{1}+2 \alpha_{2}+3 \alpha_{3}+4 \alpha_{4}+3 \alpha_{5}+2 \alpha_{6}+\alpha_{7}
\end{gathered}
$$

and the conclusion follows.

Besides being split or anisotropic, the possible indexes for $G$ are given by Table 11.

\begin{tabular}{|c|cc|}
\hline $\begin{array}{c}\text { semisimple anisotropic } \\
\text { kernel of } G\end{array}$ & index of $\beta_{G}(1)$ & $r(G)$ \\
\hline$E_{6}$ & 1 & $\neq 0$ \\
$D_{6}$ & divides 4 & 0 \\
$A_{1} \times D_{5}$ & 2 & 0 \\
$A_{1} \times D_{4}$ & 2 & 0 \\
$D_{4}$ & 1 & 0 \\
$A_{1}^{\times 3}$ & 2 & 0 \\
\hline
\end{tabular}

TABle 11. Possible invariants of a group $G$ of type $E_{7}$ that is neither split nor anisotropic.

Lemma 11.3. Suppose that $G$ is as in Prop. 11.1(2). Then:

(1) $G$ is anisotropic and every extension that splits $G$ has dimension divisible by 24 .

(2) Let $K$ be an extension such that $G$ is $K$-isotropic.

(a) If 3 does not divide $[K: F]$, then $G$ has $K$-rank 1 and 8 divides $[K: F]$.

(b) If 3 divides $[K: F]$ but 4 does not, then $G$ has $K$-rank 1 and 6 divides $[K: F]$. 
Proof. Since $\beta_{G}(1)$ has index $8, G$ is anisotropic by Table 11. Further, if $G$ is split over an extension $L / F$, then $L$ must kill both $\beta_{G}(1)$ and $r(G)$, hence the dimension $[L: F]$ is divisible by 8 and 3 respectively. This proves (1).

For (2a), the hypothesis on the dimension implies that $K$ does not kill $r(G)$, hence $G \times K$ must have semisimple anisotropic kernel $E_{6}$ by Table 11 .

For $(2 \mathrm{~b})$, the hypothesis on the dimension implies that $\beta_{G}(1) \times K$ has index at least 4 , hence the semisimple anisotropic kernel of $G \times K$ must have type $D_{6}$ by Table 11 .

The lower bound "divisible by 24" in Lemma 11.3(1) is easily obtained. One might hope to prove a stronger bound, but this is impossible. For any group $G$ of type $E_{7}$, the greatest common divisor of the dimensions $[L: F]$-where $L$ ranges over extensions of $F$ splitting $G$-divides 24 by $[$ To, 6.1].

Proof of Prop. 11.1(2). Because $G$ is anisotropic and remains so over every quadratic extension of $F$ by Lemma 11.3(2a), it cannot contain a rank 1 torus nor an $F$-subgroup of type $A_{1}$. By symmetry, $G$ contains no rank 6 tori nor any semisimple subgroup of rank 6 .

Over a co-3-closure $F_{3}$ of $F$, the group $G$ has rank 1 by Lemma 11.3(2a). But a group of type $C_{3}, G_{2}, D_{n}$ for $n \neq 4, A_{n}$ for $n=3,4,6,7$, or $A_{1}^{\times s}$ for $s \neq 3$ has $F_{3}$-rank $\geq 2$. So such a group cannot be an $F$-subgroup of $G$.

A group of type $F_{4}, A_{2}$, or $A_{5}$ has rank $\geq 2$ over an extension of $F$ of degree dividing 6 , hence it cannot be an $F$-subgroup of $G$ by Lemma $11.3(2 \mathrm{~b})$.

Suppose that $H$ is a semisimple $F$-subgroup of $G$ that is not normalized by a maximal torus of $G$. Table 34 on p. 233 of [Dy] gives a list of the possibilities for the type of $H$, using the assumption that $F$ has characteristic zero. All of them have an isotypic component of type $A_{1}, G_{2}, F_{4}$, or $C_{3}$, so they cannot be $F$-subgroups of $G$ by the preceding observations.

Now suppose that $H$ is a semisimple subgroup of $G$ of type $A_{2}^{\times 2}$. We claim that its centralizer has semisimple type $A_{2}$. It suffices to prove this over an algebraic closure of $F$, where [Dy] says that all subgroup of type $A_{2}^{\times 2}$ are conjugate. In particular, we may assume that $H$ is generated by the root subgroups corresponding to the highest root, $\alpha_{1}, \alpha_{6}$, and $\alpha_{7}$ as in the extended Dynkin diagram

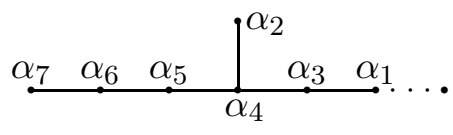

The intersection of the maximal torus $T$ in $G$ with $H$ gives a maximal torus $S$ in $H$. The centralizer $Z_{G}(S)$ is reductive with semisimple part generated by the root subgroups for roots $\alpha_{2}$ and $\alpha_{4}$. Clearly, these root subgroups centralize $H$ also, proving the claim. As $G$ has no $F$-subgroups of type $A_{2}$, this is a contradiction, so $G$ has no subgroups of type $A_{2}^{\times 2}$. 
To complete the proof of (2), we consult the list of possible semisimple subalgebras of $E_{7}$ in Table 11 in [Dy], and note that all the types other than $D_{4}, A_{1}^{\times 3}$, and $D_{4} \times A_{1}^{\times 3}$ have rank 6 or have an isotypic component excluded by the previous arguments.

11.5. Suppose now that $G$ is a group of type $E_{7}$ over a field $F$ of characteristic $\neq 2$, and that $G$ contains a semisimple subgroup of type $D_{4} \times A_{1}^{\times 3}$. The $A_{1}^{\times 3}$ component is isogenous to $R_{L / F}(\mathrm{SL}(Q))$ for some cubic étale $F$-algebra $L$ and quaternion $L$-algebra $Q$, and the Tits algebra of $G$ is Brauer-equivalent to $\operatorname{cor}_{L / F}[Q]$. To see this, we examine the center of the $A_{1}^{\times 3}$ component in case $G$ is split. Viewing $A_{1}^{\times 3}$ as generated by the roots from (11.2), the nonidentity elements of the center of each copy of $A_{1}$ are

$$
h_{\alpha_{7}}(-1), \quad h_{\alpha_{2}}(-1) h_{\alpha_{3}}(-1) h_{\alpha_{7}}(-1), \quad \text { and } \quad h_{\alpha_{3}}(-1) h_{\alpha_{5}}(-1) h_{\alpha_{7}}(-1)
$$

respectively, where $h_{\alpha_{i}}: \mathbb{G}_{m} \rightarrow G$ is the cocharacter corresponding to the simple root $\alpha_{i}$. In particular, the fundamental weight $\omega_{7}$ of $G$ restricts to the product map $\mu_{2}^{\times 3} \rightarrow \mu_{2}$ on the center of $A_{1}^{\times 3}$. By [Ti 71, §5], this proves the claim.

The previous paragraph implies that the Tits algebra of every group of type $E_{7}$ over a 2-special field is Brauer-equivalent to a tensor product of 3 quaternion algebras. Indeed, it is certainly Brauer-equivalent to $Q_{1} \otimes$ cor $_{K / F}\left(Q_{2}\right)$ where $Q_{1}, Q_{2}$ are quaternion algebras over $F$ and a quadratic étale $F$-algebra $K$ respectively. But $\operatorname{cor}_{K / F}\left(Q_{2}\right)$ has degree 4 and exponent 2 , so it is isomorphic to a tensor product of two quaternion algebras.

\section{Maximal indexes of Tits algebras}

The purpose of this section is extend Theorem 12.1 to include also the most interesting cases where the character $\chi$ is not fixed by $\operatorname{Gal}(F)$. Specifically, we prove:

Theorem 12.1. $\quad$ (1) A superversal group of type ${ }^{2} A_{n-1}$ for $n$ odd is isogenous to $\mathrm{SU}(D, \tau)$ for $D$ a division algebra of degree $n$ with unitary involution $\tau$. Further, $\lambda^{k} D$ has index $\left(\begin{array}{l}n \\ k\end{array}\right)$ for $1 \leq k \leq n$.

(2) A superversal group $G$ of type ${ }^{2} E_{6}$ has Tits algebra $\beta_{G}(1)$ of index 27.

(3) A superversal group $G$ of type ${ }^{3} D_{4}$ or ${ }^{6} D_{4}$ has Tits algebras $\beta_{G}(\chi)$ of index 8 for $\chi \neq 0$.

To prove Theorem 1.1, we only need the result for type ${ }^{3} D_{4}$. The result for type ${ }^{6} D_{4}$ is known (and we provide here a different proof); it is Prop. 8.1 in [KPS].

The theorem follows easily from Lemma 12.5, stated and proved below.

12.2. We now set up the proof of Lemma 12.5. Fix a root system $\Phi$, a set of simple roots $\Delta$, a subgroup $\Gamma$ of $\operatorname{Aut}(\Delta)$, and a dominant minuscule weight $\lambda$. Write $G^{d}$ for the split simply connected group with root system 
$\Phi$ and $\left(I_{\mu}, \rho_{\mu}\right)$ for the irreducible representation of $G^{d}$ with highest weight $\mu$. Define a representation $\left(V^{d}, \rho^{d}\right)$ of $G^{d}$ by setting

$$
V^{d}:=\bigoplus_{\mu \in \Gamma \cdot \lambda} I_{\mu} \otimes I_{\mu}^{*}
$$

as a vector space, where $G^{d}$ acts on $I_{\mu}$ via $\rho_{\mu}$ and acts trivially on $I_{\mu}^{*}$. As a representation of $G^{d}$, we have:

$$
\left(V^{d}, \rho^{d}\right) \cong \bigoplus_{\mu \in \Gamma \cdot \lambda} \bigoplus^{\operatorname{dim} I_{\mu}}\left(I_{\mu}, \rho_{\mu}\right)
$$

We also define a representation $\rho^{A}: \operatorname{Aut}_{\Gamma}\left(G^{d}\right) \rightarrow \operatorname{GL}\left(V^{d}\right)$ for $\operatorname{Aut}_{\Gamma}\left(G^{d}\right)$ as in Def. 5.2. The identity component of $\operatorname{Aut}_{\Gamma}\left(G^{d}\right)$ is the adjoint group, and it acts via the natural representation on $I_{\mu}$ and $I_{\mu}^{*}$. (The representations $\rho_{\mu}$ and $\rho_{\mu}^{*}$ of $G^{d}$ do not factor through the adjoint group, but $\rho_{\mu} \otimes \rho_{\mu}^{*}$ does.) For each $\gamma \in \Gamma$, we have $\rho_{\mu}\left(\gamma^{-1} g\right)=\operatorname{Int}(a) \rho_{\gamma \mu}(g)$ for some matrix $a$ depending on $\gamma$ and $\mu$. Combining these matrices with the natural permutation representation on the weights in $\Gamma \cdot \lambda$, we find an action of $\operatorname{Aut}_{\Gamma}\left(G^{d}\right)$ on $V^{d}$. Furthermore, for $\alpha \in \operatorname{Aut}_{\Gamma}\left(G^{d}\right)$ and $g \in G^{d}$, we have:

$$
\rho^{d}(\alpha g)=\operatorname{Int}\left(\rho^{A}(\alpha)\right) \rho^{d}(g) .
$$

Now fix a 1-cocycle $z \in Z^{1}\left(F, \operatorname{Aut}_{\Gamma}\left(G^{d}\right)\right)$. We twist $G^{d}$ and $\rho^{d}$ by $z$ to obtain a group $G={ }_{z} G^{d}$ and a representation $\rho: G \rightarrow{ }_{z} \operatorname{GL}\left(V^{d}\right)$ because by Hilbert 90 theorem we have an isomorphism $z \operatorname{GL}\left(V^{d}\right) \cong \mathrm{GL}\left(V^{d}\right)$.

More concretely, $G$ is the same as $G^{d}$ over $F_{\text {sep }}$, but the Galois group $\operatorname{Gal}(F)$ acts on $G\left(F_{\text {sep }}\right)$ via the action ${ }_{z}$ defined by $\sigma \cdot_{z} g:=z_{\sigma} \sigma(g)$. The 1-cocycle $\rho^{A}(z)$ takes values in $\operatorname{GL}\left(V^{d}\right)$, and so is $\sigma \mapsto h^{-1 \sigma} h$ for some $h \in \mathrm{GL}(V)\left(F_{\text {sep }}\right)$. We put: $\rho:=\operatorname{Int}(h) \rho^{d}$. Then for $g \in G\left(F_{\text {sep }}\right)$, we have:

$$
\begin{aligned}
\sigma \rho(g) & =\operatorname{Int}(\sigma h) \rho^{d}(\sigma g)=\operatorname{Int}(h) \operatorname{Int}\left(\rho^{A}\left(z_{\sigma}\right)\right) \rho^{d}(\sigma g) \\
& =\operatorname{Int}(h) \rho^{d}\left(z_{\sigma} \sigma g\right)=\rho\left(\sigma \cdot_{z} g\right),
\end{aligned}
$$

which confirms that $\rho$ is defined over $F$ [Bo, AG.14.3].

Lemma 12.5. Let $\lambda$ be a minuscule dominant weight of a root system $\Phi$ with set of simple roots $\Delta$. Fix $\Gamma \subset \operatorname{Aut}(\Delta)$. If

$$
\operatorname{gcd}\left\{\operatorname{dim} I_{\lambda},\left|Z^{*}\left(F_{\text {sep }}\right)\right|,|\Gamma \cdot \lambda|\right\}=1,
$$

then $\beta_{G}(\lambda)$ has index $n_{G}(\lambda)$ for every superversal group $G$ constructed from $\Delta$ and $\Gamma$.

We define $\beta_{G}(\lambda)$ and $n_{G}(\lambda)$ where the subscript $\lambda$ is a weight of $\Phi$ to be $\beta_{G}(\chi)$ and $n_{G}(\chi)$ (in the notation of $\S 8$ ), where $\chi$ is the restriction of $\lambda$ to the center of $G$.

Proof of Lemma 12.5. Let $H$ be a group of type $\Phi$ over a field $F$. The fundamental property we use is that there is a unique irreducible representation $J_{H, \lambda}$ of $H$ over $F$ such that the composition series for $J_{H, \lambda}$ over an algebraic 
closure of $F$ has $I_{\lambda}$ as a quotient; this follows from [Ti 71, Th. 7.2]. This representation satisfies

$$
\operatorname{dim} J_{H, \lambda}=|\operatorname{Gal}(F) \cdot \lambda| \cdot \operatorname{ind} \beta_{H}(\lambda) \cdot \operatorname{dim} I_{\lambda} .
$$

Twisting the representation $\rho^{d}$ of $G^{d}$ defined in 12.2 by a superversal torsor gives a representation $W$ of $G$, and the simple quotients in the composition series of $W$ are copies of $J_{G, \lambda}$.

We specialize the superversal group $G$ to a versal group $G^{\prime}$ of inner type defined over a field $K^{\prime}$. The representation $W$ of $G$ specializes to a representation $W^{\prime}$ of $G^{\prime}$. As $\lambda$ is fixed by $\operatorname{Gal}\left(K^{\prime}\right)$, the composition series for $W^{\prime}$ over $K^{\prime}$ has irreducible quotients various copies of $J_{G^{\prime}, \mu}$ for $\mu \in \Gamma \cdot \lambda$.

We view the irreducible quotients of the composition series as partitioning the weights of $W$, equivalently the weights of $W^{\prime}$. Because $G$-invariant subspaces of $W$ specialize to $G^{\prime}$-invariant subspaces of $W^{\prime}$, the partition coming from the composition series under $G^{\prime}$ is a refinement of the partition of the composition series under $G$. The dimension of $J_{G^{\prime}, \mu}$ depends only on the Aut $(\Delta)$-orbit of $\mu$-by (12.6) and (8.3) - so $\operatorname{dim} J_{G, \lambda}$ is a multiple of $\operatorname{dim} J_{G^{\prime}, \lambda}$. That is,

$$
\operatorname{ind} \beta_{G^{\prime}}(\lambda) \cdot \operatorname{dim} I_{\lambda} \quad \text { divides } \quad|\Gamma \cdot \lambda| \cdot \operatorname{ind} \beta_{G}(\lambda) \cdot \operatorname{dim} I_{\lambda}
$$

by (12.6). By Th. 8.7, the index of $\beta_{G^{\prime}}(\lambda)$ is the number $n_{G^{\prime}}(\lambda)$. Tracing through the definition of $n_{G^{\prime}}(\lambda)$, the hypothesis on $\lambda$ implies that ind $\beta_{G^{\prime}}(\lambda)$ and $|\Gamma \cdot \lambda|$ are relatively prime, so ind $\beta_{G^{\prime}}(\lambda)$ divides ind $\beta_{G}(\lambda)$, i.e.,

$$
n_{\lambda}\left(G^{\prime}\right)=\operatorname{ind} \beta_{G^{\prime}}(\lambda)\left|\operatorname{ind} \beta_{G}(\lambda)\right| n_{G}(\lambda) .
$$

Since the numbers $n_{G^{\prime}}(\lambda), n_{G}(\lambda)$ depend only on the root system, they are equal, and this proves the lemma.

\section{Groups of TYPE ${ }^{3} D_{4}$ or ${ }^{6} D_{4}$}

Let $G$ be a simple group of type $D_{4}$ over $F$. The cocenter of its simply connected cover is $\mathbb{Z} / 2 \mathbb{Z} \times \mathbb{Z} / 2 \mathbb{Z}$ with a possibly nontrivial Galois action, and $n_{G}(\chi)$ is 8 for $\chi \neq 0$.

Lemma 13.1. Suppose that $G$ is a group of type ${ }^{1} D_{4},{ }^{3} D_{4}$ such that $\beta_{G}(\chi)$ has index 8 for $\chi \neq 0$. Then:

(1) $G$ is anisotropic.

(2) If $K / F$ is a finite extension of $F$ of dimension not divisible by 8, then the $K$-rank of $G$ is $\leq 1$.

Proof. (1) is known. For $G$ of type ${ }^{3} D_{4}$, it is [Ga 98, 2.5], and for type ${ }^{1} D_{4}$ it is even easier.

For (2), suppose first that $G$ has type ${ }^{1} D_{4}$, i.e., that $G$ is isogenous to $\mathrm{SO}(D, \sigma, f)$ for some central division $F$-algebra of degree 8 with quadratic pair such that the even Clifford algebra $C_{0}(D, \sigma, f)$ is isomorphic to $A \times B$ for $A$ and $B$ central division $F$-algebras of degree 8 . By the hypothesis on 
$K$, the index of $A \otimes K, B \otimes K$, and $D \otimes K$ is in all cases at least 2. It follows that the involution $\sigma$ is not $K$-hyperbolic [KMRT, 8.31], which is the claim.

For $G$ of type ${ }^{3} D_{4}$, there is a unique cubic Galois extension $L / F$ over which $G$ is of type ${ }^{1} D_{4}$. The compositum $K L$ has dimension $[K L: L]$ dividing $[K: F]$, and in particular $[K L: L]$ is not divisible by 8 . Replacing $F$ and $K$ with $L$ and $K L$, we are reduced to the type ${ }^{1} D_{4}$ case.

Lemma 13.2. Let $G$ be a group of type ${ }^{3} D_{4}$ or ${ }^{6} D_{4}$ over a field of characteristic $\neq 3$. If $r(G) \neq 0$, then $G$ is anisotropic.

The hypothesis on the characteristic is here so that $r(G)$ is defined, cf. Prop. 6.2. Recall from Table 6 that $r(G)$ is an element of $H^{3}(F, \mathbb{Z} / 3 \mathbb{Z})$. If $G$ has type ${ }^{1} D_{4}$ (or ${ }^{2} D_{4}$ ), then $r(G)$ is automatically zero.

Proof of Lemma 13.2. $G$ is not quasi-split; suppose it is isotropic. The paper [Ga98] gives a description of $G$ by Galois descent (which also works in characteristic 2 with only cosmetic changes), and in particular $G$ is quasisplit by a separable quadratic extension $K$ of the field. As $K$ kills $r(G)$ and the dimension of $K$ is not divisible by $3, r(G)$ is zero.

Theorem 13.3. Let $G$ be a group of type ${ }^{3} D_{4}$ over a field of characteristic $\neq 3$. If the Tits algebras $\beta_{G}(\chi)$ have index 8 for nonzero $\chi$ and $r(G)$ is not zero, then every nonzero proper reductive subgroup of $G$ is a maximal torus.

Proof. By Lemma 13.2, $G$ is anisotropic and remains anisotropic over a co3 -closure of $F$. It follows that $G$ does not contain a rank $1 F$-torus nor a rank $2 F$-torus split by an extension of degree dividing 8. By Lemma 13.1, $G$ contains no rank $2 F$-tori split by an extension of degree dividing 12 . By symmetry and Table 3 , every nonzero $F$-torus in $G$ is maximal.

Over an algebraic closure, a proper semisimple subgroup of $G$ of rank 4 has type $A_{1}^{\times 4}$. An $F$-group of this type becomes isotropic over a separable extension of $F$ of dimension a power of 2, hence by Lemma 13.2 cannot be contained in $G$.

The hypothesis on $r(G)$ was used to exclude transfers of groups of type $A_{1}$ from a quartic field extension $L / F$. These are genuine dangers: The paper [CLM] shows that every isotropic group of type ${ }^{3} D_{4}$ or ${ }^{6} D_{4}$ contains a subgroup isogenous to $R_{L / F}\left(\mathrm{SL}_{2}\right)$.

We close by proving the theorem stated in the introduction.

Proof of Th. 1.1. Let $G$ be a superversal group of type ${ }^{3} D_{4}$ over a field $F$ of characteristic $\neq 2,3$ (itself an extension of some base field $F_{0}$ ). Prop. 12.1 gives that $\beta_{G}(\chi)$ has index 8 for nonzero $\chi$. Because a superversal group is in particular a versal group, we have $r(G) \neq 0$ by Lemma 6.5. Then $G$ is almost abelian by Th. 13.3 and 2.5 .

As for type ${ }^{6} D_{4}$, since there are almost abelian groups of type ${ }^{3} D_{4}$ defined over a suitable extension of $F_{0}$, Corollary 5.5 implies that the superversal group $G$ of type ${ }^{6} D_{4}$ is almost abelian. 
Acknowledgments. We thank Gopal Prasad for useful conversations about the global field case. The first author's research was partially supported by National Science Foundation grant DMS-0654502. He thanks the Institut des Hautes Etudes Scientifiques for its hospitality while the work on this article was done.

\section{REFERENCES}

[Atlas] J. H. Conway, R.T. Curtis, S.P. Norton, R. A. Parker, R. A. Wilson, Atlas of finite groups, Oxford University Press, Eynsham, 1985.

[BdS] A. Borel and J. De Siebenthal, Les sous-groupes fermés de rang maximum des groupes de Lie clos, Comment. Math. Helv. 23 (1949), 200-221.

[Bo] A. Borel, Linear algebraic groups, second ed., Graduate Texts in Mathematics, vol. 126, Springer-Verlag, New York, 1991.

[BoTi] A. Borel and J. Tits, Éléments unipotents et sous-groupes paraboliques de groupes réductifes. I, Invent. Math. 12 (1971), 95-104.

[Bou] N. Bourbaki, Lie groups and Lie algebras: Chapters 4-6, Springer, 2002.

[C] V. Chernousov, The kernel of the Rost invariant, Serre's Conjecture II and the Hasse principle for quasi-split groups ${ }^{3,6} D_{4}, E_{6}, E_{7}$, Math. Ann. 326 (2003), 297-330.

[CS] V. Chernousov and J.-P. Serre, Lower bounds for essential dimensions via orthogonal representations J. Algebra 305 (2006), 1055-1070.

[CLM] V. Chernousov, L. Lifschitz, and D.W. Morris, Almost-minimal nonuniform lattices of higher rank, preprint, 2007.

[Da] E.C. Dade, The maximal subgroups of $4 \times 4$ integral matrices, Illinois J. Math. 9 (1965), 99-122.

[Dy] E.B. Dynkin, Semisimple subalgebras of semisimple Lie algebras, Amer. Math. Soc. Transl. (2) 6 (1957), 111-244, [Russian original: Mat. Sbornik N.S. 30(72) (1952), 349-462].

[Ga 98] S. Garibaldi, Isotropic trialitarian algebraic groups, J. Algebra 210 (1998), 385418.

[Ga] Cohomological invariants: exceptional groups and spin groups, with an appendix by Detlev W. Hoffmann, Memoirs Amer. Math. Soc., to appear.

[GaQ] S. Garibaldi and A. Quéguiner-Mathieu, Restricting the Rost invariant to the center, Algebra i Analiz 19 (2007), 52-73, reprinted as St. Petersburg Math. J. 19 (2008), 197-213.

[Gi] P. Gille, Invariants cohomologiques de Rost en caractéristique positive, $K$-Theory 21 (2000), 57-100.

[GiS] P. Gille and T. Szamuely, Central simple algebras and Galois cohomology, Cambridge studies in Advanced Math., vol. 101, Cambridge, 2006.

[KMRT] M.-A. Knus, A.S. Merkurjev, M. Rost, and J.-P. Tignol, The book of involutions, Colloquium Publications, vol. 44, Amer. Math. Soc., 1998.

[K] M. Kneser, Lectures on Galois Cohomology of the Classical Groups, Tata Institute of Fundamental Research lectures in mathematics and physics 47(1969), Tata Institute of Fundamental Research.

[KP] J. Kuzmanovich and A. Pavlichenkov, Finite groups of matrices whose entries are integers, Amer. Math. Monthly 109 (2002), no. 2, 173-186.

[KPS] M.-A. Knus, R. Parimala, and R. Sridharan, On generic triality, Algebra, arithmetic and geometry, Part I, II (Mumbai, 2000), Tata Inst. Fund. Res. Stud. Math., vol. 16, Tata Inst. Fund. Res., Bombay, 2002, pp. 355-404.

[Mer 96] A.S. Merkurjev, Maximal indexes of Tits algebras, Doc. Math. J. DMV 1 (1996), 229-243. 
[Mer 03] _ Rost invariants of simply connected algebraic groups, with a section by S. Garibaldi, in "Cohomological invariants in Galois cohomology", University Lecture Series, vol. 28, Amer. Math. Soc., 2003.

[MPW1] A.S. Merkurjev, I. Panin, and A.R. Wadsworth, Index reduction formulas for twisted flag varieties, I, K-Theory 10 (1996), 517-596.

[MPW2] _ , Index reduction formulas for twisted flag varieties, II, K-Theory 14 (1998), no. 2, 101-196.

[NSW] J. Neukirch, A. Schmidt, K. Wingberg, Cohomology of number fields, Grundlehren der Mathematischen Wissenschaften 323 (2000), Springer-Verlag, Berlin.

[PIR] V. Platonov and A. Rapinchuk, Algebraic groups and number theory, Academic Press, 1994.

[PrR 86] G. Prasad and A. Rapinchuk, Computation of the metaplectic kernel, Publications Mathématiques de l'IHES 84 (1996), 91-187.

[PrR 08] G. Prasad and A. Rapinchuk, Local global principles for embedding of fields with involution into simple algebras with involutions, preliminary version, 17 Jan. 2008.

[Se 92] J-P. Serre, Lie algebras and Lie groups, 2nd ed., Lecture Notes in Math., vol. 1500, Springer-Verlag, 1992.

[Se 02] , Galois cohomology, Springer-Verlag, 2002, originally published as Cohomologie galoisienne (1965).

[Se 03] Cohomological invariants, Witt invariants, and trace forms, notes by S. Garibaldi, in "Cohomological invariants in Galois cohomology", University Lecture Series, vol. 28, Amer. Math. Soc., 2003.

[SGA3] M. Demazure and A. Grothendieck, Schémas en groupes, Lecture Notes in Mathematics, vol. 151-153, Springer, 1970.

[Sp] T.A. Springer, Linear algebraic groups, second ed., Birkhäuser, 1998.

[St] R. Steinberg, Torsion in reductive groups, Adv. Math. 15 (1975), no. 1, 63-92 [= Collected Papers, pp. 415-444].

[Ta] K.-I. Tahara, On the finite subgroups of $\mathrm{GL}_{3}(\mathbb{Z})$, Nagoya Math. J. 41 (1971), 169-209.

[Ti 66] J. Tits, Classification of algebraic semisimple groups, Algebraic Groups and Discontinuous Subgroups, Proc. Symp. Pure Math., vol. IX, AMS, 1966, pp. 32-62.

[Ti 71] _ Représentations linéaires irréductibles d'un groupe réductif sur un corps quelconque, J. Reine Angew. Math. 247 (1971), 196-220.

[Ti 87] Unipotent elements and parabolic subgroups of reductive groups. II, Algebraic groups (Utrecht, Netherlands, 1986), Lecture Notes in Math., vol. 1271, Springer, 1987, pp. 33-62.

[Ti 91] R Résumé des cours de 1990-91, Annuaire du Collège de France (1991), $125-137$

[Ti 92a] Résumé des cours de 1991-92, Annuaire du Collège de France (1992), $115-133$

[Ti 92b] Sur les degrés des extensions de corps déployant les groupes algébriques simples, C. R. Acad. Sci. Paris Sér. I Math. 315 (1992), no. 11, 1131-1138.

[To] B. Totaro, The torsion index of $E_{8}$ and other groups, Duke Math. J. 129 (2005), 219-248. 
Department of Mathematics \& Computer Science, Emory University, AtLANTA, GA 30322, USA

E-mail address: skip@member.ams.org

$U R L:$ http://www . mathcs . emory.edu/ skip/

UMR 8552 Du CNRS, DMA, École normale supérieure, F-75005 PARIs, France

E-mail address: Philippe.Gille@ens.fr 